\title{
A Review of Embedded Large Eddy Simulation for Internal Flows
}

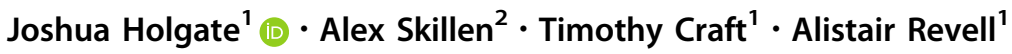

Received: 31 January 2018 / Accepted: 6 May 2018/Published online: 18 May 2018

(C) The Author(s) 2018

\begin{abstract}
When scale-resolving simulation approaches are employed for the simulation of turbulent flow, computational cost can often be prohibitive. This is particularly true for internal wall-bounded flows, including flows of industrial relevances which may involve both high Reynolds number and geometrical complexity. Modelling the turbulence induced stresses (at all scales) has proven to lack requisite accuracy in many situations. In this work we review a promising family of approaches which aim to find a compromise between cost and accuracy; hybrid RANS-LES methods. We place particular emphasis on the emergence of embedded large eddy simulation. These approaches are summarised and key features relevant to internal flows are highlighted. A thorough review of the application of these methods to internal flows is given, where hybrid approaches have been shown to offer significant benefits to industrial CFD (relative to an empirical broadband modelling of turbulence). This paper concludes by providing a cost-analysis and a discussion about the emerging novel use-modalities for hybrid RANS-LES methods in industrial CFD, such as automated embedded simulation and multi-dimensional coupling.
\end{abstract}

\section{Introduction}

The investigation and understanding of wall bounded turbulent flows is of great importance to both academic and industrial institutions, with many practical applications ranging from aerospace to nuclear power. The understanding of flow phenomena such as thermal fatigue in T-juctions [27], traverse vibration due to generation of Dean vortex [21] switching in pipe elbows [127] and the application of ribbed ducts to enhance heat transfer [64] have benefitted from CFD of one form or another for several decades now. A commonly used approach in industrial CFD involves solving the discretised Reynolds averaged Navier-Stokes (RANS) equations, with closure

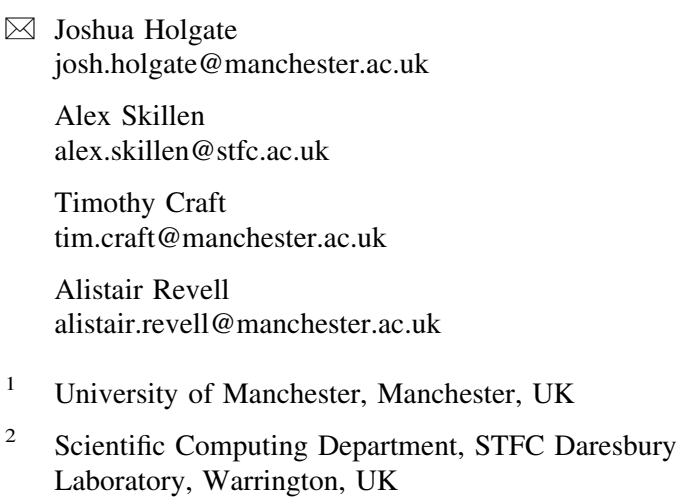

provided by a turbulence model. This approach allows for reduced grid resolution; particularly within the near wall regions. While this approach allows relatively cheap simulations, the accuracy limitations of RANS methods can't be ignored.

Direct numerical simulation (DNS) entails resolution of the full bandwidth of turbulence, and thus eliminates the accuracy issues of RANS. However, this imposes substantial demands upon grid resolution and overall costs. Large eddy simulation (LES) alleviates some of the expense associated with DNS by removing the computation of the smallest, dissipative scales that account for $99 \%$ of the overall computation cost [74]. Sub Grid Scale (SGS) turbulence models, first introduced by Smagorinsky [89], account for the effect of the small scale structures whilst the large scales of motion are resolved. Despite this, sufficient computational power is still unavailable to match the high resolution demands required for wall resolved LES for complex cases at high Reynolds Numbers.

Spalart [91] suggests the readiness of fully resolved LES is still a considerable time away for simulations of complex geometry. Recent studies have suggested a deceleration in Moore's law. Moreover Waldrop [119] states that the growth factor of Moore's law has slowed greatly with current computing techniques reaching their maximal operating limits. This was more recently emphasised by Larsson and Wang [52] who, based on recent 
technological advancements, projected that LES will not, and simply cannot, replace RANS solely in the design process within the next 30 years. Instead Larsson suggests it is more appropriate to consider questioning how and where LES could be utilised in conjunction with RANS methods within the design process.

It is these considerations that have led to growing recognition for the coupling of RANS and LES, in attempt to unite the advantages of each method [14]. Models of this nature have been generalised as hybrid RANS LES Methods (HRLM), with one prominent subcategory being Embedded LES - where regions of LES are embedded within a broader RANS domain (Fig. 1)

Despite LES being deemed more accurate than RANS, it does not mean that this accuracy is guaranteed. The sensitivity of LES imposes a well defined QA criteria that stipulates a strong dependence on numerics and grid generation, and it is possible that a weakly prescribed LES can produce worse results than a standard RANS. This conditioning is arguably controlled by the expertise and experience of the user. The risks associated with low experience users could potentially be alleviated by adopting an autonomous/semi-autonomous CFD approach. and it is argued that such software could become as significant as the improvement of computational speeds within industrial design [52].

The purpose of the following paper is to highlight the challenges associated with modelling internal turbulent flows, notably turbo-machinery and nuclear components found within industry; and to explore the potential benefits that hybrid RANS-LES methods can provide. Through initially presenting the currently available approaches of hybrid methods whilst also addressing the notable limitations on interface coupling, the advantages of the hybrid approaches becomes clear, with a comprehensive review of their application to industry style cases being presented. Furthermore comments on the cost perspective of both LES and hybrid RANS-LES approaches is given in Sect. 4, which provides a distinct outlook on the benefits to be gained. The paper concludes by introducing two new approaches to CFD within industry,

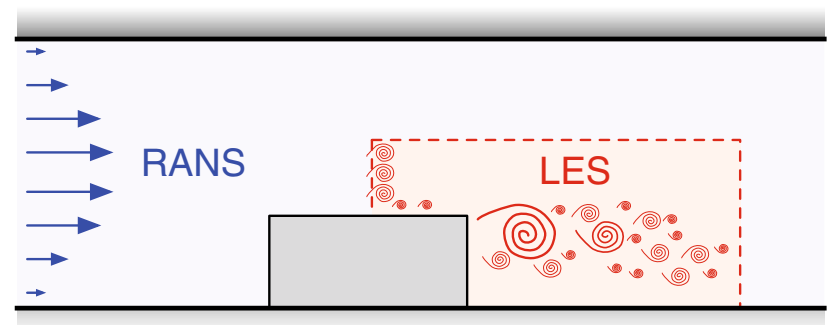

Fig. 1 Example of embedded LES zonal arrangement with RANS/ LES coupling aimed at progressing the continuing development and readiness of high accuracy computational methods outside of academia.

\section{Hybrid RANS-LES}

For even relatively simple geometries, the use of LES for internal flow is restricted to moderate Reynolds numbers, due to the mesh requirements in the near wall region. HRLMs can generally be separated into two distinct classes: (1) Global and (2) Zonal and shall be briefly introduced in the following section. The reader is also briefly referred to Table 1 for a simple comparison between the two classes.

\subsection{Global RANS-LES}

Global approaches are based on the continuous treatment of flow variables at model defined interfaces [25] which are generally prescribed from the solution. These methods follow a unified approach and generally solve one set of transport equations throughout the entire domain. An alternate formulation for the turbulent length scale forces a switch between the RANS and LES, which typically occurs within the near wall regions.

A widely used global method is detached eddy simulation (DES), originally proposed two decades ago by Spalart [94]. DES is a single grid approach where an unsteady RANS simulation, originally the Spalart-Allmaras model [92] but later adapted for others such as $k-\omega S S T[59,95]$ and the elliptic relaxation $\varphi-f$, is solved globally with a switching term enabling the model to change to LES when the local turbulent length scale exceeds the local grid dimensions [94]. DES has been implemented with great success for a number of different applications, whilst generally performing better for flows dominated by large regions of separation. A wide range of successful studies employing DES have been reported in the literature, beyond the scope of the present work; instead the reader is encouraged to refer to the following examples $[15,56,101,113]$. This approach does however suffer from a couple notable issues as described by Deck [23], Tucker and Davidson [105] and Spalart et al. [93] (amongst others). Most notably amongst these is Modelled Stress Depletion (MSD) and Grid Induced Separation (GIS), where inconsistent handling between the filtered LES and time-averaged stresses, which are assumed equal at the RANS - LES interface, can lead to a reduction in the skin friction and ultimately initiates premature separation. It is documented that this issue is typically a result of an unwanted switch to LES within an attached boundary layer due to local grid refinement. 
Table 1 Advantages and disadvantages of global and zonal approaches to hybrid RANS-LES

\begin{tabular}{lll}
\hline Hybrid approach & Advantages & Disadvantages \\
\hline Global & Limited user input required & Grey area problem and Log-layer mismatch \\
& Good for globally and locally unstable flows & Modelled stress depletion (MSD) \\
& Relatively easy to implement & Grid induced separation (GIS) \\
& Simple to use & Delay in formation of instabilities \\
& Single mesh used globally & Struggle in stable flow regimes i.e. pipe flows \\
Gonal & Grid refinement only in regions of interest & Increased user decision load \\
& No delay in formation of instabilities & A priori knowledge of flow is favourable \\
& Fixed interfaces removes risk of MSD and GIS & Synthetic turbulence required at R-L interface \\
& Can be employed for stable flow computations & Must re-establish statistical data at L-R interface \\
\hline
\end{tabular}

The more recently developed Delayed DES (DDES) by Spalart et al. [93] and later Improved DDES (IDDES) of Shur et al. [86], use blending functions to prevent the early switching to LES in attached boundaries. Meanwhile IDDES further extends the model's capabilities by combining the approach with WMLES to resolve the mismatch between the modelled and resolved log layers.

In addition to DES, there has been a number of global methods developed over the past decade which have no explicit definition of the RANS-LES interface, instead are based upon a modified RANS model that adapts to scale resolving simulation under specific conditions. Such examples of this class include the Partially Averaged Navier-Stokes (PANS) [30] and the Partially Integrated Transport Method (PITM) [10]. The former uses a sensor based upon the ratio of modelled to total turbulent kinetic energy to determine which turbulence solving mode the model acts. The PANS ratio was later improved by Basara et al. [8] to allow for run time adaptation.

An alternate approach to the general consensus of global methods has been proposed by Xiao and Jenny [125], in the form of a consistent dual-mesh hybrid RANS - LES, with the model more recently being generalised and applied to wall-bounded turbulent flow by Tunstall et al. [108]. Although not strictly of the natural form of the global methods the duel-mesh approach solves both RANS and LES models simultaneously on separate grids, with added drift terms in the momentum equation used to relax the flow statistics towards a consistent solution, or that which is the most relevant at a particular space and time. This framework alleviates the fundamental problems encountered along the interfaces in general global methods such as DES, allowing a clean solution between near-wall RANS and LES free-stream conditions. In a similar approach Uribe et al. [110], a two-velocity field splits the residual stress tensor into two parts, locally isotropic and inhomogeneous, with a blending function between the two.

\subsection{Zonal RANS-LES}

Restricting LES to a specific portion of the domain, whilst maintaining a RANS solution elsewhere offers potential for gains in efficiency. Generally, zonal approaches adopt segregated LES regions embedded within a RANS solution. Each subdomain is then solved individually with a separate set of transport equations. For example, to a tworegion Embedded LES simulation is shown in Fig. 2; this figure illustrates the transition region between an upstream RANS and downstream LES through the injection of synthetic fluctuations. Unlike global methods, there is a clear transitional boundary between RANS and LES regions, although this imposes further complications which shall be considered later.

As an extension to DDES, Deck proposed a zonal formulation of the standard model [22, 23]. Zonal DES (ZDES) acted to utilise a-priori knowledge of the flow field in order to strategically prescribe specific RANS and DES regions in the domain. The method proved advantages for flows characterised by strongly developing turbulence inherited from upstream boundary layers. ZDES differs from Spalart's DES through the definition of a ZDES length scale, sub-grid scale length and treatment of the near wall function in LES mode [50]. Improved formulations of ZDES were later released again by Deck [24, 26].

Poletto et al. [72] investigated the use of Embedded DDES regions whilst computing flow around a 2D hump, with an emphasis on a synthetic RANS to LES interface condition. They noted a considerable reduction in CPU time between an embedded DDES approach and a full domain DDES method could be achieved, with an overall $30 \%$ speed-up in CPU requirement per time step.

In addition Davidson and Peng have worked towards producing a PANS based ELES model [17, 18] again with an emphasis on the interface conditions, where Partially Averaged Navier-Stokes (PANS) is a modified $k-\epsilon$ model that can operate in both RANS and LES modes. 
Fig. 2 Example of a two region embedded LES pipe simulation showing the transition between RANS and LES (left), using the field of 3-axis ellipsoid synthetic eddies randomly generated at the RANS-LES interface represented as a schematic (right)

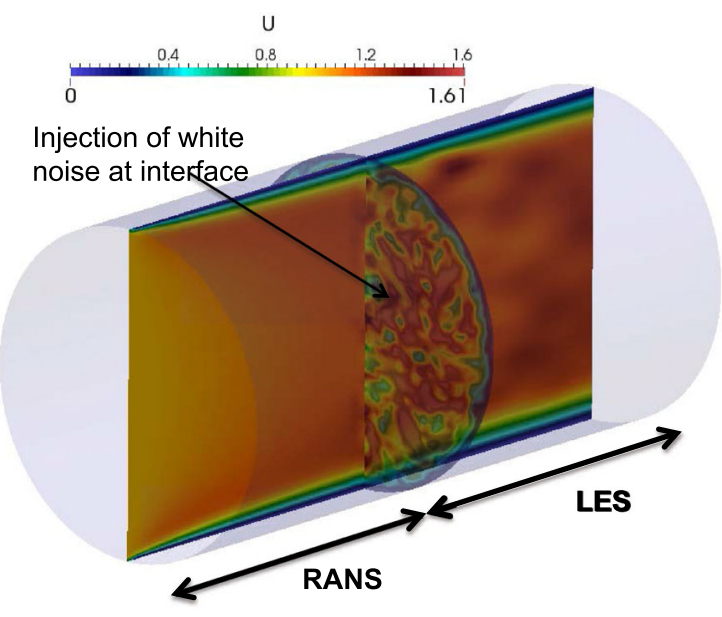

However the studies indicate that the results within the LES region are highly sensitive to the interface conditions and upstream turbulent quantities, with the recovery of the recirculation regions downstream of the hump flow slightly over-predicting the measured value.

An early implementation of ELES is presented by Cokljat et al. [14] who considered a fully developed pipe, channel flow and flow over a backwards-facing step. This work was one of the first to demonstrate the potential of ELES whilst offering notable advantages to industrial CFD. A number of more recent implementations have followed, most notably $\mathrm{Li}$ et al. al. [54] validated a tworegion ELES solver that consisted of single RANS and implicit LES zones with the transfer of variables handled at the interface. They applied this implementation to both channel flow and flow over periodic hills noting good agreement to full LES data. Anupindi and Sandberg [2] implemented an ELES model within OpenFOAM based again on separate regions for the RANS and LES zones and the transfer of flow variables via the interfaces. In a slightly different approach, Vonlanthen et al. [118] produced a one-way nesting procedure which embeds a highly resolved LES within a low resolution LES.

The original concept for embedded simulation arose broadly from the need of LES users to define unsteady inlet boundary conditions from mean flow data, but the challenges facing a more general use of ELES are significantly greater. In theory the LES region itself could be entirely engulfed within a global RANS region, as depicted in Fig. 1. For instance a cuboidal LES region with 6 sides will have a different combination of inlet and outlets depending on the flow direction, and in more complex flows, these definitions may change during the simulation; it is indeed quite likely that instantaneous flow will travel in both directions across any boundary [4]. It is these interfacing features which represent the majority of new developments in the community, along with the development of best practice guidelines to facilitate it's usage [60].

\subsubsection{RANS to LES Interface}

Tabor and Baba-Ahmadi [96] presented a comprehensive review on the subject of inlet conditions for LES, separating the methods into two distinct categories; (1) Precursor/recycling methods and (2) Synthesised Methods. The former involves computing a precursor simulation either prior to or concurrently with the target LES to generate a library of turbulent content that can then be mapped, and scaled if required, onto the inlet of the LES domain. In contrast, synthesised methods involve the injection of synthetic fluctuations upon a prescribed mean flow. More recently $\mathrm{Wu}$ [124] provided an updated review on LES inlet techniques, whilst also beginning to describe their application within an hybrid RANS-LES framework. Following the classification proposed by Tabor, Wu highlights that research has led to the emergence of several synthetic techniques. Wu argues that recycling methods are less efficient than the synthetic counterparts and despite being highly accurate their additional costs and lack of generality restricts use with hybrid models [39].

The easiest approach would be to inject white noise upon the mean flow of the upstream RANS. Although simple this method fails to provide the spatial or temporal correlations required to sustain turbulent generation downstream, as shown by Schluter et al. [78] and illustrated in Fig. 3 (top). Here the generated velocity field disappears very quickly with the flow immediately becoming laminar.

In attempt to alleviate the lack of spatial and temporal coherence Jarrin et al. [38] presented the Synthetic Eddy Method (SEM). SEM is derived from the vortex method originally outlined in a $\mathrm{PhD}$ thesis by Sergent [81]. Unlike white noise, SEM generates a fluctuation field through the 

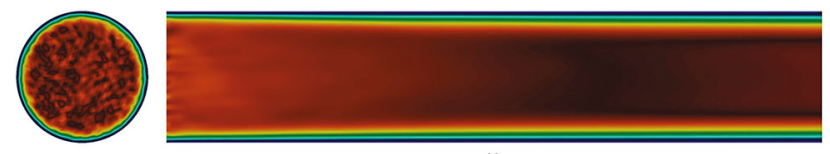

0.4 0

0.8
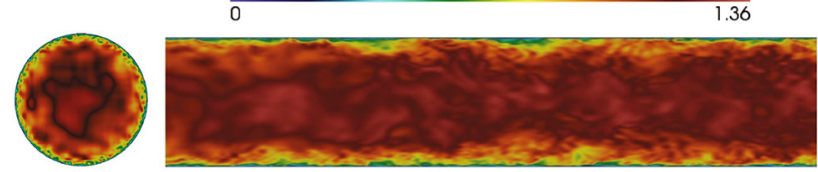

Fig. 3 Comparison between the flow development within an LES pipe using different synthetic inlet conditions, images taken from a slice of both the inlet plane and stream-wise centreline. (top) White noise inlet. (bottom) Improved synthetic Eddy method

superposition of coherent structures across an allotted plane, each with a prescribed weighted velocity distribution. The generated velocity fluctuations are normalised to reproduce the required second order turbulent statistics, based upon an upstream RANS field, via a Cholesky decomposition of the Reynolds stress tensor.

Improvements to the SEM formulation have since materialised, aiming to reduce the recovery/development length of the resolved turbulence whilst potentially alleviating some of the computational costs. Firstly an improved version by Adamian et al. [1] documented a decrease in error of the averaged flow parameters whilst also shortening the transition region, through a modification of the determining of the linear scale of generated eddy structures. Meanwhile Skillen et al. [88] proposed an alternate fluctuation normalisation factor, based upon a running average of the eddy concentration that guarantees the desired statistical properties, regardless of the spatial distribution or length-scale of the eddies, thus correctly allowing for an inhomogeneous distribution of eddy size. Figure 3 (bottom) illustrates the generated velocity field within OpenFOAM acting on a straight pipe flow. The computation here is one-way coupled such that the instantaneous turbulent fluctuations are superimposed onto a mean velocity profile obtained from an upstream Reynolds Stress Model (RSM).

Pamies et al. [66] also provided subsequent improvements to the original SEM formulation by modifying the eddy shape function to take into account the variance in structures across the turbulent boundary layers observed in experiments. Each randomly generated eddies' shape, length and time scales are dependable on their 'altitude' or distance from the wall, and are adjusted depending on the specification of its given mode value.

Meanwhile the most recent improvements to SEM incorporates the use of anisotropic forcing of the Reynolds Stresses within an overlapping region of RANS and LES. This method was originally outlined in a PhD thesis by De Meux et al. [19] although the reader is also directed to a later publication by the same author [20], both documenting a decrease in development length and a faster recovery of the turbulent statistics.

Despite positive results perhaps the most documented inherent weakness of the various forms of SEM is that the generated velocity field does not satisfy the divergence-free condition, leading to an introduction of significant pressure fluctuations at the inlet plane [71]. This can have a detrimental effect upon the development length, and expectedly pressure fluctuations are unwanted when one is concerned with noise prediction. Divergence Free SEM (DFSEM) is an alternative version to the standard approach that proposed a change in definition of the velocity fluctuations associated with the eddies, to provide a divergence free velocity field. Poletto et al. [73] reports the development of DFSEM, and is largely based on the original methodology by Jarrin et al. [38], however instead of applying the fluctuations to the velocity field directly they are first applied to the vorticity field and then transformed back to velocity by taking the curl of the vorticity.

In a separate category to the synthetic eddy variants there exists a range of synthetic turbulence models based upon the superposition of weighted spatiotemporal Fourier model, reviewed in detail by Tabor and Baba-Ahmadi [96]. A notable example developed for specific use in hybrid RANS-LES is the NTS Synthetic Turbulence Generator (STG), documented by Shur et al. [87].

Similarly Auerswald and Bange [5] developed an anisotropic synthetic generator based on probability of Fourier modes. This approach was later extended by Auerswald et al. [6] to account for the integral length scales in $y$ and $z$ directions.

Additionally a divergence free approach to Fourier mode analysis has been published by Patruno and Ricci [69].

Despite the somewhat documented difficulty and importance of a well developed synthetic fluctuation method for the inlet to LES simulation (both coupled and uncoupled RANS-LES), the recent publications reviewed above are proving to be reasonbaly successful. Both the NTS STG and SEM variants have been proven to produce 'life-like' turbulence consistent with the desired statistics, whilst also maintaining a short development/lag phase with minimal computational expense.

As the case with ELES it may be required that the computation switch back to RANS mode from an upstream LES. Thus introducing another interface with differing demands to those when passing from RANS to LES. A review and explanation of the methods involved is provided in the next section. 


\subsubsection{LES to RANS Interface}

On returning to the RANS domain it is required that one recovers the mean flow data from the resolved turbulent descriptions produced from LES. Thus the interface must aim to imitate a statistical averaging operation, where the LES solution is averaged both spatially and temporally to remove the unnecessary turbulent data. Additionally the interface must ensure that the fluctuations from the LES solution leaving the LES domain do so without incorrectly producing turbulent anomalies in the RANS domain [46]. This averaging process takes place at the interface which itself houses an overlapping region between the two meshes [79], this enables for flow variables to spatially adapt to the model change, whilst trilinear interpolation is used to exchange flow quantities between meshes.

However the process is not as trivial when reestablishing the modelled turbulent quantities, since these don't appear within the LES formulation. Instead values such as eddy viscosity, turbulent kinetic energy and turbulent frequency have to be reconstructed. Konig et al. [46] introduced three different methods to accomplish this, testing each case upon a turbulent channel flow. Each method involves calculating the turbulent kinetic energy directly from the LES solution as, $k=\frac{1}{2} u_{i}^{\prime \prime} u_{i}^{\prime \prime}$ for the turbulent viscosity, method $\mathrm{A}$ and $\mathrm{B}$ firstly determines the turbulent dissipation, $\epsilon$ from the transport equation of the turbulent kinetic energy with method A taking into account both the resolved and unresolved scales whilst method B neglects the unresolved motions. Method C takes a slightly different approach, and instead calculated the turbulent viscosity via computing the turbulent frequency, $\omega$. This approach (method $\mathrm{C}$ in particular) has recently been utilised by Anupindi and Sandberg [2] for use in an ELES solver implemented in OpenFOAM with reasonable success documented. Meanwhile Gritskevich et al. [34] document the results of two different approaches to the recovery of the mean flow data when passing from LES to RANS. The first, labelled a 1 stage approach continuously solves the chosen RANS model throughout the whole computational domain. However, within the LES region the RANS model runs passively in the background using the LES flow field but having no effect upon the solution; upon entering the RANS domain the solution to the RANS model is reinstated. The second or 2 stage approach initially computes the whole domain with the RANS model, the solution for the turbulent variables i.e. turbulent kinetic energy $(\mathrm{k})$, turbulent dissipation $(\epsilon)$ are then frozen within the LES domain during the second computation. This frozen solution is then re-activated at the inlet to the RANS domain.

\section{HRLM Applied to Internal Flows}

In the following section we review examples of (1) the benefits of using LES over RANS, and (2) the application of hybrid RANS-LES methods, both in the context of internal flows. All the papers discussed in this section are summarised in Table 2 for clarity and to facilitate comparison.

\subsection{Turbo-Machinery}

\subsubsection{Internal Cooling Ducts}

Turbine blades are generally operating at temperatures above the operational limit of the blade material, and small fluctuations above the design temperature can reduce a systems operational life by $50 \%$ [111]. These facts would suggest that the cooling mechanism employed is of the upmost importance. Cooling is achieved through internal ducts housed within the blade and employ forced convection through induced separation, promoting highly turbulent/unsteady flow [114]. The flow characteristics and prediction of heat transfer have been extensively studied on these geometries using RANS, LES and increasingly hybrid RANS-LES. In addition the angle alignment and size of the ribs have also been altered in attempt to assess the variation in heat transfer capabilities.

Table 2 documents a number of the reviewed application computations, evidently many are available for internal cooling systems/ribbed ducts. The RANS studies listed report incorrect predictions of the Nusselt number [90] demonstrating a variation of around 250\% [102]. Tucker [104] provided a compilation of some of the more notable RANS models' prediction of Nusselt number distribution across a ribbed channel, see Fig. 4. In this case it is likely that diffusion terms in the RANS models would act to suppress the high levels of near wall turbulence, having a direct impact on the heat transfer in this region.

The relatively poor performance of RANS models is not entirely unexpected, particularly their application to complex three-dimensional structures or regions of flow separation. Heat transfer is directly linked to the structure of the flow, and so if the turbulence is not correctly captured then one cannot expect to calculate the correct levels of heat transfer.

Increased accuracy of these flows are achieved when adopting LES. Tafti [97] conducted a computation of a periodic ribbed duct at a Reynolds number 20,000, using a relatively large grid resolution both the heat transfer and skin friction coefficient was predicted within $5 \%$ of experimental values. However the computation is then 
Table 2 Summary of industrial relevant flow applications reviewed in this paper and the modelling method applied, also shown are the grid densities and Reynolds number used for each case respectively

\begin{tabular}{|c|c|c|c|c|}
\hline Application & Author(s) & Modelling method & $R e_{b} / 10,000$ & No. grid points $/ 10^{6}$ \\
\hline Channel flow & Hoyas and Jimenez [36] & DNS & 9 & $1.8 \times 10^{4}$ \\
\hline Channel flow & Pantano et al. [67] & LES & 9 & 5.7 \\
\hline Channel flow & Kalitzin et al. [43] & Hybrid & 9 & 0.27 \\
\hline Ribbed duct & Tafti [97] & LES & 2 & 2.1 \\
\hline Ribbed duct & Sewall and Tafti [83] & LES & 2 & 8.9 \\
\hline Ribbed duct & Labbe [49] & LES & 4 & 5.2 \\
\hline Ribbed duct & Sewall et al. [84] & LES & 2 & 8.85 \\
\hline Ribbed duct & Lohasz et al. [57] & LES & 4 & 0.47 \\
\hline Ribbed duct & Viswanathan and Tafti [115] & Hybrid & 2 & 0.26 \\
\hline Ribbed duct & Viswanathan and Tafti [113] & Hybrid & 2 & 0.26 \\
\hline Ribbed duct & Liu et al. [56] & Hybrid & 1.42 & 0.45 \\
\hline Ribbed duct & Kubacki [47] & Hybrid & 2 & 2 \\
\hline Ribbed duct & Ooi et al. [64] & RANS & 3 & 0.18 \\
\hline Ribbed duct & Kamali [44] & RANS & 2 & 0.13 \\
\hline Ribbed duct & Xie et al. [126] & RANS & 6 & 2 \\
\hline Ribbed duct & Sohankar [90] & 2DRANS & 10 & 0.015 \\
\hline Two-pass cooling duct & Sewall and Tafti [82] & LES & 2 & 8.4 \\
\hline Two-pass cooling duct & Tyache and Tucker [109] & Hybrid & 2 & 7 \\
\hline Two-pass cooling duct & Viswanathan and Tafti [116] & Hybrid & 2 & 7.7 \\
\hline Two-pass cooling duct & Schuler et al. [80] & Hybrid & 2.5 & 5.8 \\
\hline Two-pass cooling duct & Tucker [103] & Hybrid & 2 & 7 \\
\hline T-junction & Kuczaj et al. [48] & LES & 9.5 & 7.3 \\
\hline T-junction & Lee et al. [53] & LES & 9.5 & 1.3 \\
\hline T-junction & Ndombo and Howard [62] & LES & 9.5 & 10 \\
\hline T-junction & Obabko [63] & LES & 40 & 21 \\
\hline T-junction & Aulery et al. [7] & LES & 40 & 2.2 \\
\hline T-junction & Chapuliot et al. [12] & VLES & 15 & 0.2 \\
\hline T-junction & Westin et al. [122] & Hybrid & 47 & 0.39 \\
\hline T-junction & Zeng and Li [128] & Hybrid & 47 & 0.39 \\
\hline T-junction & Ming and Zhao [61] & Hybrid & 9 & 4.9 \\
\hline T-junction & Gritskevich et al. [32] & Hybrid & 2.8 & 2.8 \\
\hline T-junction & Gritskevich et al. [32] & Hybrid & 9 & 4.9 \\
\hline T-junction & Walker et al. [120] & RANS & 9 & 0.78 \\
\hline T-junction & Aounallah et al. [3] & URANS & 7 & 0.58 \\
\hline T-junction & Frank et al. [27] & URANS & 19 & 0.5 \\
\hline T-junction with bend & Pasutto [68] & LES & 1 & 0.85 \\
\hline T-junction with bend & Aulery et al. [7] & LES & 100 & 4.8 \\
\hline Pipe flow & Tavakoli et al. [100] & LES & 2 & 1.1 \\
\hline Pipe flow & Ould-Rouiss et al. [65] & LES & 0.5 & 0.26 \\
\hline Wall mounted cube & Jrgensen [41] & Hybrid & 13 & 5.47 \\
\hline Plane diffuser & Fadai-Ghotbi et al. [37] & Hybrid & 1 & 1.86 \\
\hline Asymmetric diffuser & Davidson [16] & Hybrid & 1.8 & 0.52 \\
\hline
\end{tabular}

much more costly with almost 20 hours required to compute a single flow through.
In addition, Labbe [49] (conducting a study based on the experimental set-up by Casarsa et al. [9]) noted that the LES flow filed is strongly linked to the inlet conditions 
Fig. 4 Nusselt number, $\mathrm{Nu}$ distribution and subsequent heat transfer capability across a ribbed channel for various RANS models reproduced from $[56,104]$ (left), and instantaneous streamlines in side and top down views for Zonal LES computation from [56] (right)

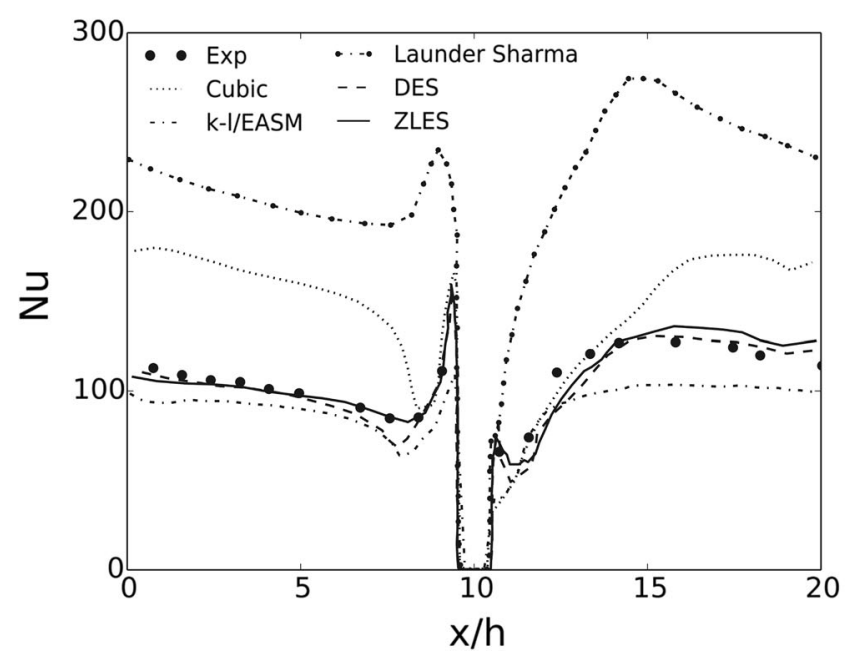

applied. Here slight discrepancies were found due to a poor handling of the inlet.

As yet there are, to our knowledge, no published documents using ELES for an internal cooling duct. Instead most have chosen to adopt global hybrid methods, most notably DES. However in a similar context Jorgensen et al. [42] applied ELES to flow around a wall-bounded cube. A schematic of the domain is shown in Fig. 5. Synthetic fluctuations in the form of the 2D vortex method of Mathey al el. [58] were applied at the inlet to the LES domain. The mean and standard deviations of the streamwise flow component showing good agreement with experimental data and also the body induced turbulence

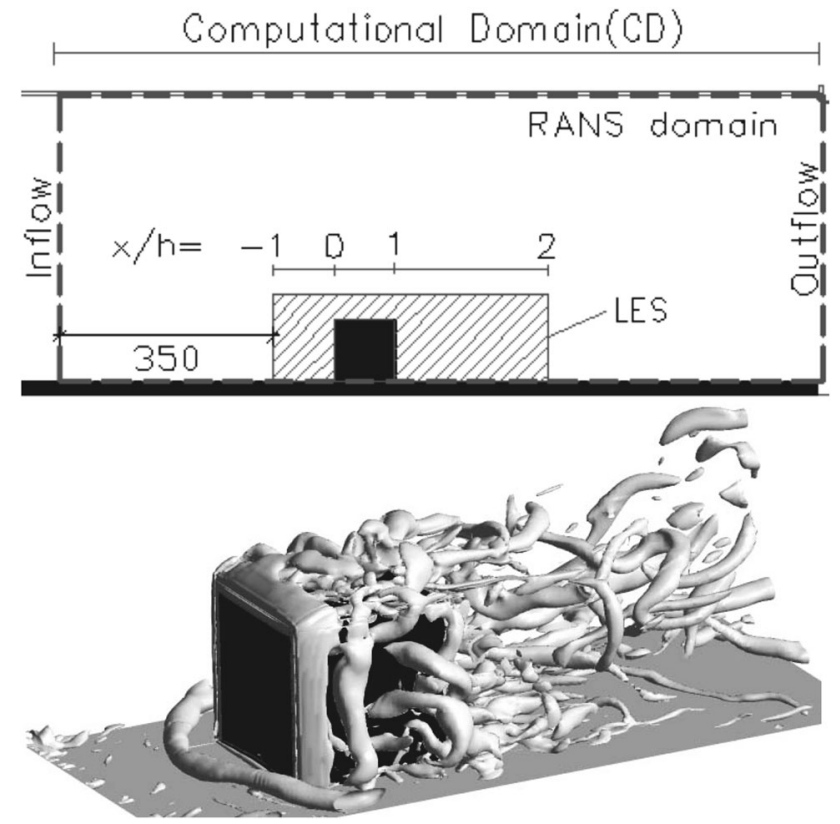

Fig. 5 (top) Schematic of the embedded LES domain for the floormounted cube used by Jorgensen et al. [42]. (bottom) Coherent small scale vortex structures generated within the LES domain [42] being well captured, despite this the simulations experienced some difficulties due to poor control of the upstream turbulent structures.

In order to mitigate the high costs reported above, Viswanathan and Tafti [113] adopted DES on the same domain used by Tafti [97] achieving only a $9 \%$ deviation with respect to fine grid LES at $1 / 10$ th of the cost. Additionally several studies by Vishwanathan and Tafti [112, 115, 117], Liu et al. [56] and Kubacki et al. [47] successfully implemented hybrid based methods to predict the turbulent and mean flow features in stationary, rotating and roughened ribbed ducts - all documenting reasonable success.

Again Tucker [104] has compiled a comparative graph of the Nusselt number development across a single rib for a number of turbulence models, including hybrid RANSLES show in Fig. 4 (right). It is shown that the hybrid models provide a much more favourable result than any RANS alternative, despite slight over-predictions estimated by ZLES after re-attachment of the flow.

One could argue that global hybrid methods may be more suitable for flows of this nature given the proximity of subsequent ribs, where a clear interface cannot be defined for ELES as the high accuracy detail is required throughout the whole domain, rather than within a particular confinement of the geometry.

\subsubsection{Two-Pass Cooling Duct}

This case is an extension of the the single periodic ribbed duct flow including two channels connected via a $180^{\circ}$ bend. Sewall and Tafti [82] conducted an LES study of a stationary two pass duct, with and without a rib in the bend, whilst Viswanathan et al. [116] conducted the same simulation using a $k-\omega$ based DES model. The former LES study required a grid resolution of 0.7 million cells greater 
than the DES of Viswanathan et al. nearly an order of magnitude less than Sewall's simulation. Despite the DES computation over-predicting the flows developments length at the inlet there is a good quantitate comparison with both LES and experimental results.

\subsection{Nuclear Applications}

The development and operation of nuclear reactors demand accurate understanding of the complex thermal hydraulics involved in order to ensure rigorous safety regulations are satisfied. For example, excessive thermal fatigue resulting from the turbulent mixing of fluids at differing temperatures can lead to a complete destruction of the system [12]. It is argued that thermal fatigue is the most common cause of unexpected failures within Nuclear Power Plants (NPP's) particularly within piping components such as mixing tee's [29]. Flows throughout complex piping geometries are highly unsteady and involve broad bandwidth, low frequency fluctuations that are poorly represented through standard URANS [31]. Westin et al. [122] and Walker et al. [120] illustrated that both steady and unsteady RANS calculations are unable predict realistic mixing between two flows [122].

Additional structural vibration and fluid-born noise can be generated in pipe regions involving sharp bends due to the presence of large pressure gradients [129]. The capturing of these secondary flow features has been studied extensively by Takamura et al. [98], Yuki et al. [127], Tanaka and Ohshima [99] amongst others for the Japan Sodium cooled Fast Reactor (JSFR).

The complex and transient characteristics of the flow regimes found in the nuclear industry exemplifies the requirement for higher order turbulence models within constraints the constraints of resources.

\subsubsection{Thermal Mixing in a T-Junction}

A number of reports have successfully adopted LES to the problem of thermal mixing in a T-junction. Lee et al. [53] studied the temperature fluctuations and structural response of a standard mixing tee configuration by coupling LES with a newly developed thermal stress fatigue analyses. Meanwhile Ndombo and Howard [62] coupled LES with the inclusion of synthetic turbulence at the inlet. In addition to standard T-junction flows model multiple studies have also looked at the effects of an upstream pipe bend prior to the mixing zone. Pasutto et al. [68], Aulery et al. [7] and Tunstll et al. [107] successfully applied LES to predict the effects of upstream bends on the mixing of the two flow streams. All showed good agreement to experimental data whilst the later also adopted the use of DFSEM at the inlet to the two pipe streams.
Despite the obvious accuracy advantages provided from LES models their applicability within an industrial framework is limited. The computations disclosed in the previous paragraph were expensive and required access to significant High Performance Computing (HPC) resource. It can be estimated that an LES simulation for a straight inlet T-Junction at a moderate Reynolds number of $10^{4}$ with a maximum grid resolution of 20 million required, at minimum, $>300$ cpu-hours [63] to compute; with this increasing further if higher order statistics are desired. In contrast a RANS computation could be completed in less than one day, whilst still providing accurate predictions of the time-averaged thermal-hydraulic characteristics [55]. It is not uncommon for flows in nuclear applications to reach Reynolds numbers of $10^{6}$ or $10^{7}$, and so measures must be taken to reduce the cost overheads whilst maintaining a noteworthy degree of accuracy.

Gritskevich et al. [31] investigated the application of hybrid RANS-LES approaches for predicting heat transfer at the walls in a T-junction's mixing zone. Of particular interest here is the success of ELES, with Fig. 6 illustrating a typical domain decomposition for ELES in a T-Junction. In Gritskevich's computation there are two RANS to WMLES interfaces, both have been treated with the vortex method, whilst a single WMLES to RANS interface downstream of the mixing zone has no additional treatments.

It is suggested by Gritskevich et al. [31] that the use of global schemes, such as Scale Adaptive Simulation (SAS) and DDES, could be problematic for the computation of flows where there is no natural geometrical or flow induced conversion to Scale Resolving Simulation (SRS) - i.e. there is no seperated flow. This issue is highlighted by Deck et al. [26] who describes the different modes of separated flows for DES and uses this as a justification for the development of ZDES. More concisely, global models will suffer greatly if the initial instabilities of the flow aren't sufficiently strong or, if there is little variation in turbulent length and time scales across the domain. Menter et al. [60] has previously emphasised this, stating that flows deemed to be stable are characterised by a continuous development of turbulence and are largely dependent upon the upstream flow conditions; this is typical of majority of channel and pipe networks, where the flow is likely to remain attached over much of the domain. The use of Embedded LES is therefore more promising than global type models for these types of flow. The rigid interface to the LES allows for the injection of turbulent fluctuations to encourage a change to SRS, whereas there exists an ambiguity in the transfer to SRS in DES variants.

Turbulent instabilities can be subdued by numerical dissipation, and so a poor choice of convection scheme can 
Fig. 6 (top) Decomposition of computational domain for ELES T-junction calculations. (bottom) Iso-surfaces coloured with velocity, with $\mathrm{BCD}$ boundary conditions [31]
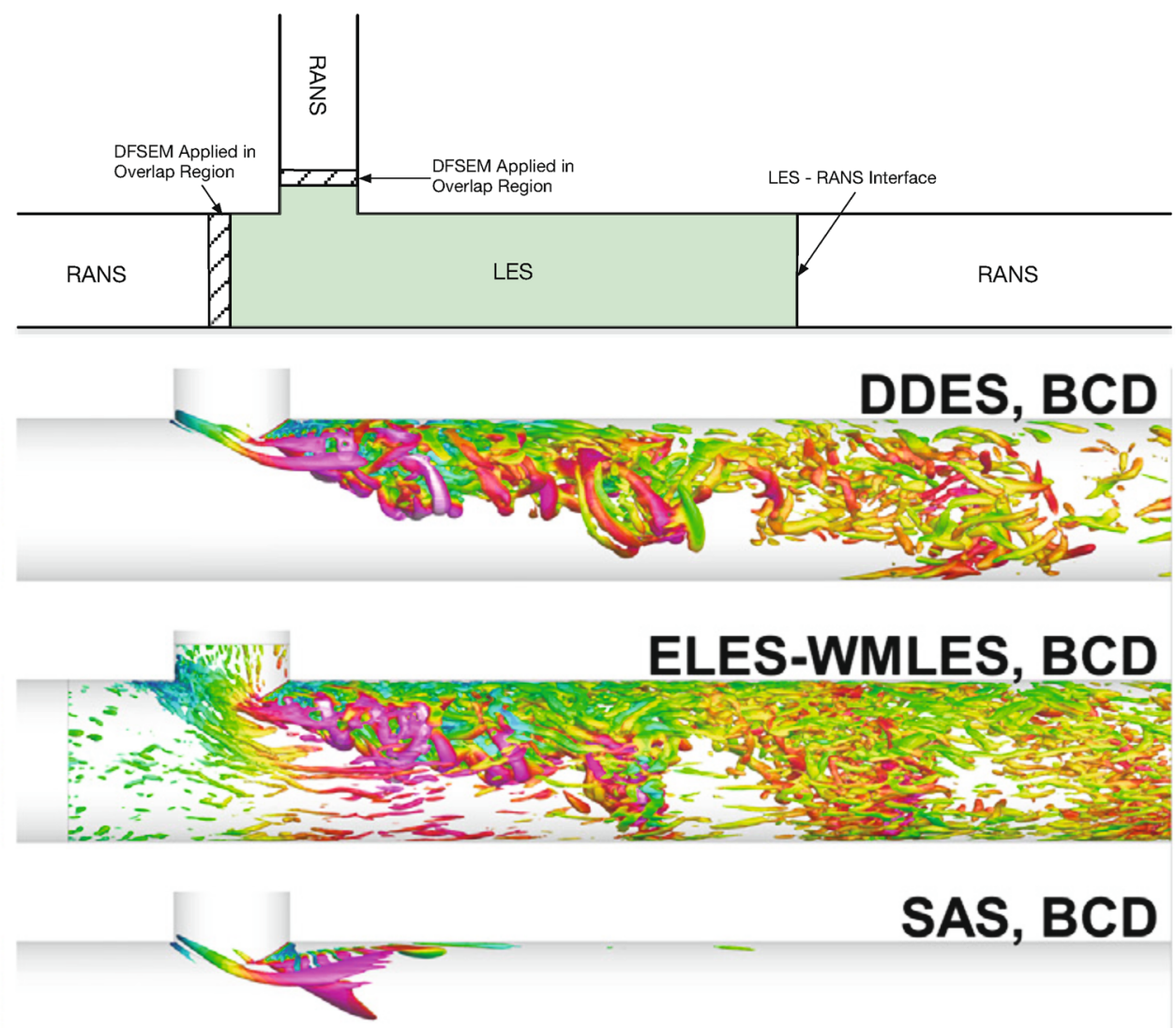

SAS, BCD

have a particularly damning effect on the level of unsteadiness in the flow. This is apparent in Fig. 6, where the dissipative nature of the second order Boundary Central Difference (BCD) scheme [40] impedes upon the formation of turbulence on both global models. However, the most striking effect occurs with SAS. A high sensitivity to the choice of velocity interpolation scheme causes a conversion back to a URANS formulation shortly downstream of the junction. This subsequently leads to a drastic underrepresentation of the RMS velocity magnitudes. On the contrary, the ELES result appears to be little affected by the convection scheme, and instead the introduction of resolved turbulence at the RANS-LES boundaries is accounted for downstream at the formation of new turbulence in the mixing zone.

The work by Gritskevich et al. [31] is later extended to include the combination of ELES with IDDES and SAS models so that the previous WMLES zones are occupied with either IDDES or SAS [33]; additionally the RANS to SRS interface is computed with both vortex method and a Generator of Synthetic Turbulence (GST) [1]. Each method was able to predict the flow and thermal mixing with reasonable accuracy with respect to experimental data, however they appear to slightly under perform when compared to the ELES case in Gritskevich et al. [31], which employed a WMLES model, albeit at additional cost. Overall the findings of the two papers for ELES is encouraging.

\subsection{Capturing Secondary Flow Structures in Pipe Bends}

T-junctions represent only one component of an entire complex piping system of a nuclear power plan or gas networks. Pipe systems commonly include a number of 90 degree bends to redirect the flow or to artificially enhance the erosion process in helium stratification [45, 77]. Although common, the flow characteristics of a 90 degree bend typically includes the pressure-induced separation at the apex, which is shown to be sensitive to the location of the upstream conditions. The separation can lead to a strong unsteady shear layer and a significant momentum deficit [77]. The flow exhibits several other distinguishing features, including: longitudinal streamline curvature, velocity profile inhomogeneity and development of secondary, swirling motions named Dean vortices [21]. These features present a significant challenge to RANS models. 
Despite this, the study of flow around a 90 degree pipe elbows has predominantly adopted stead-state RANS models. A few recent publications such as Rohrig et al. [77] have begun to embrace the application of scale resolving methods such as LES. Rohrig's study involved the benchmarking of a number of RANS models against LES for the domain seen in Fig. 7. Ultimately the study confirmed the overwhelming superiority of LES. It is found that the selected RANS models fail to correctly capture the mean velocity profile within the momentum deficit region of the bend, resulting in a underestimation in wall shear stress. It should however be noted that more favourable performance is possible when using more advances RANS models, such as the more recently developed Elliptic Blending Reynolds Stress Model (EBRSM) [51], as evidenced in the work by Tunstall et al. [106]. That said, significant deviations in the mean flow profiles remain even with EBRSM.

If employing LES for this flow, for example, at a reasonable Reynolds number of $10^{4}$, one requires approx. 20 million cells [77] to compute the flow around the bend, whereas only 1.5 million might be required to obtain a grid-converged RANS solution [106]. Another key point to note is the inlet condition. For the LES by Rohrig a fully developed profile at the inlet was applied through an a-priori LES of a straight pipe section, which itself required a computational domain of 5 million cells. The LES requires HPC to compute. Thus the costs associated with computing even a simple pipe elbow with LES is formidable for industry.

An possible alternative here is Hybrid RANS-LES or WMLES, as Tunstall et al. [106] also presented. An example of ELES was presented by Holgate et al. [35], where a reduced upstream domain size was presented as shown in Fig. 7. It can be seen that results even with an inlet length equal to 1 diameter provide good results; significantly reducing the cost of LES simulation for this flow and removing the requirement of a precursor computation entirely.

\section{Cost Perspective and Emerging Use Modes}

It is possible to identify an order-of-magnitude cost associated with internal flow simulations in terms of the spatial and temporal resolution requirements, and extrapolate this as a function of the flow Reynolds number. Figure 8 is an adaptation of cost estimates for large eddy simulation based on approximations proposed by Piomelli and Balaras [70] and Tucker [104]; largely based on the findings of Chapman [11]. The dashed line represents the LES grid estimation and is suggested to scale with $R e^{2 / 5}$.
Meanwhile the dash-dot line, scaled with $R e^{9 / 5}$, represents the wall-modelled LES with resolution down to $y+<100$. A more recent paper by Choi and Moin [13], who revisited the work by Chapman, suggests that theses relationships are much more severe i.e. scaling with $R e^{13 / 7}$ and $R e$ for wall-resolved and wall-modelled LES respectively. Approximations therein provide a broad understanding of the grid size $N$ required for conventional finite-difference/ finite-volume approaches for a range of Reynolds numbers. While loosely factored into the formulation, elements such as geometry complexity and physical domain size are not explicitly considered, and thus the estimate is taken only as a rough rule of thumb. A comparison of computational requirement for a pipe flow at a Reynolds number of around $10^{6}$ is provided in Table 3 .

As discussed in the previous section it is not uncommon for the Reynolds numbers to reach $10^{6}-10^{7}$ for industrial cases. This would suggest that grid sizes for LES computations would exceed 1 billion cells in the higher Reynolds range, which is beyond that generally available for research studies, let alone industrial work. Clearly then, this cost could be reduced substantially if a hybrid scheme was adopted, for example with a $\operatorname{Re}$ of $10^{7}$ the estimated number of nodes for WMLES is between $10^{7}$ and $10^{8}$, placing the notional computational expenses within a more realistic region. Also listed in Fig. 8 are the estimated notional cost of calculations on ARCHER, the UK's national HPC facility, ${ }^{1}$ for completing a computation with respect to the corresponding grid resolution.

A chapter by Fureby written in 'Whither Turbulence and Big Data in the 21st Century?' [28] discusses the increasing need for LES in engineering flows, but highlights the challenge posed by the generation of extremely large datasets such as those from LES/DNS of high Reynolds number flows. As an example, consider the pipe bend simulation of Rohrig et al. [77], reviewed in Sect. 3.3. Using 23 million cells the resulting memory required to store both the flow ( $u, p$ and $k$ ) and statistical variables is around 2.3 GB per time step. A typical time step is $1.5 \mathrm{~ms}$ and 6000 time-steps required per flow through time unit, with over 30 such units needed to obtain time-averaged data; which translates to around $1.8 \times 10^{5}$ time-steps. For memory and running averaging purposes each case is run with a purged timestep that provides 20 time-steps to be stored. In this case, a total memory of around $46 \mathrm{~GB}$ purely for the data would be required, (plus additional storage for the mesh and case files). This storage requirement would be expected to increase dramatically upon post-processing, for instance if high quality images and animations are required. Delving

\footnotetext{
1 ARCHER kAU and Notional cost calculator http://www.archer.ac. uk/access/au-calculator/.
} 


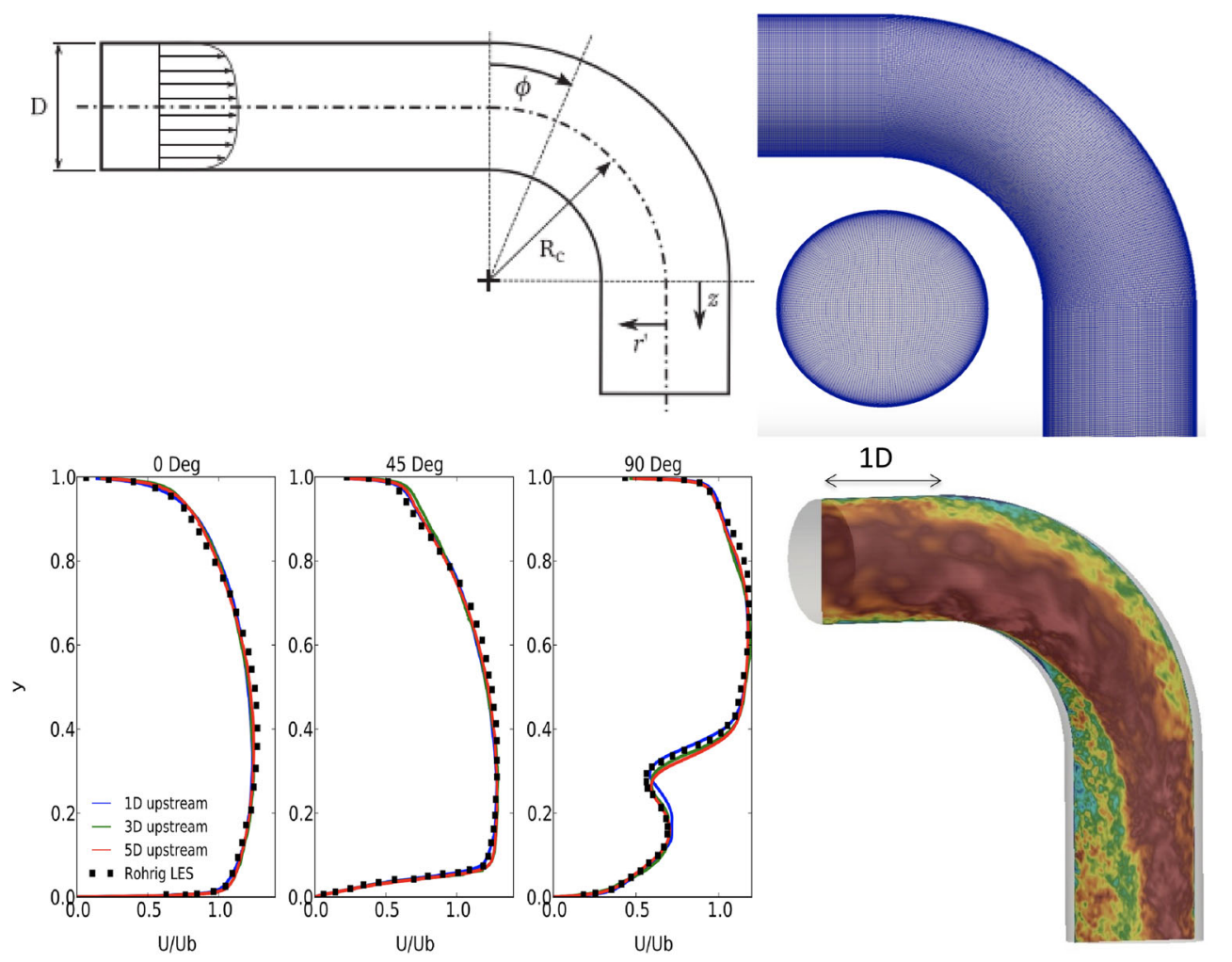

Fig. 7 Schematic of the 90 degree pipe domain of Rohrig et al. [77] (top left) and snapshot of the computational domain both across the bend and cross-section views (top right). Results for reduced inlet size

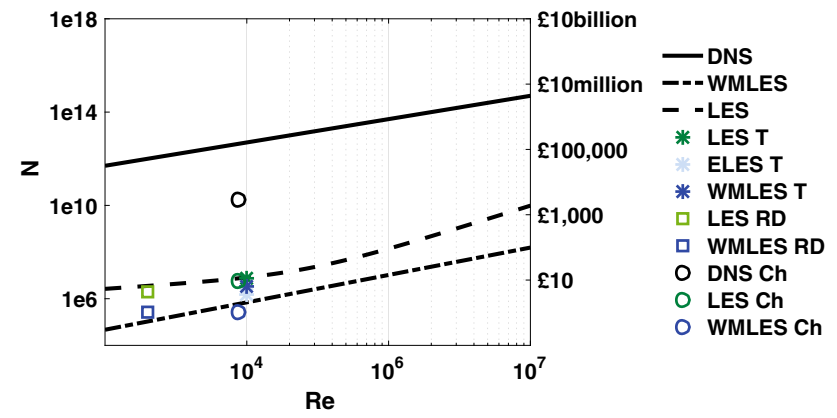

Fig. 8 Grid requirements for LES, DES and ELES with differing applications listed. Costs estimated based upon usage of UKs largest HPC facility as a non-contributing member. $\mathrm{T}=\mathrm{T}$-junction; $\mathrm{RD}=$ ribbed duct; $\mathrm{Ch}=$ channel flow

Table 3 Estimation of CPU resources required for RANS, hybrid and wall resolved LES for internal flow applications i.e. pipe flow at $R e \approx 10^{6}$

\begin{tabular}{llll}
\hline & No. of cells & No. of time steps & Cost relative to RANS \\
\hline URANS & $10^{5}-10^{6}$ & $10^{2}-10^{3}$ & 1 \\
Hybrid & $\approx 10^{7}$ & $10^{5}$ & $10^{3}$ \\
LES & $\approx 10^{8}$ & $10^{5} 6-10^{6}$ & $10^{4}-10^{5}$ \\
\hline
\end{tabular}

Method based on current meshing guidelines of LES region (btm left) and instantaneous velocity for case with 1D upstream inlet section (btm right)

deeper into the running of this simulation, it was found that on 18 nodes ( 432 processors, $\approx 55000$ cells per processor), $\approx 5 s$ real-time was required per time-step. Therefore to complete the case and allow for higher order statistics to be correctly averaged then a total run-time would exceed 10 days, costing in excess of 1.5 MAUs. Such resources will remain beyond the reach of a large section of industrial CFD users for some time to come.

A more recent alternative to in-house commercial CFD involves the outsourcing of larger computations to online or cloud based services. Wu et al. [123] illustrates the advantages gained from using cloud based environments. A good overview on the definition, characteristic and overall potential impact of cloud computing can be found in a publication by Shawish and Salama [85].

\subsection{Automated Embedded Simulation}

In light of what has been presented in this paper, and more importantly the information learned from previous studies surrounding the application of hybrid RANS-LES models, a workflow can be proposed for Embedded Simulation. A semi-automating capability is highly attractive for industry, based on the use of RANS as a precursor to ELES, where the users input and required knowledge is substantially 
Fig. 9 Flowchart for automating the embedded simulation process
STEP 1: Precursor RANS
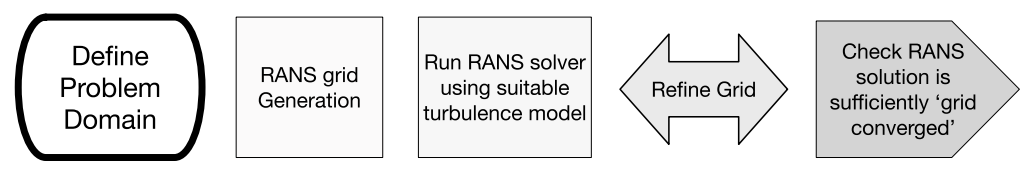

STEP 2: Embedded LES

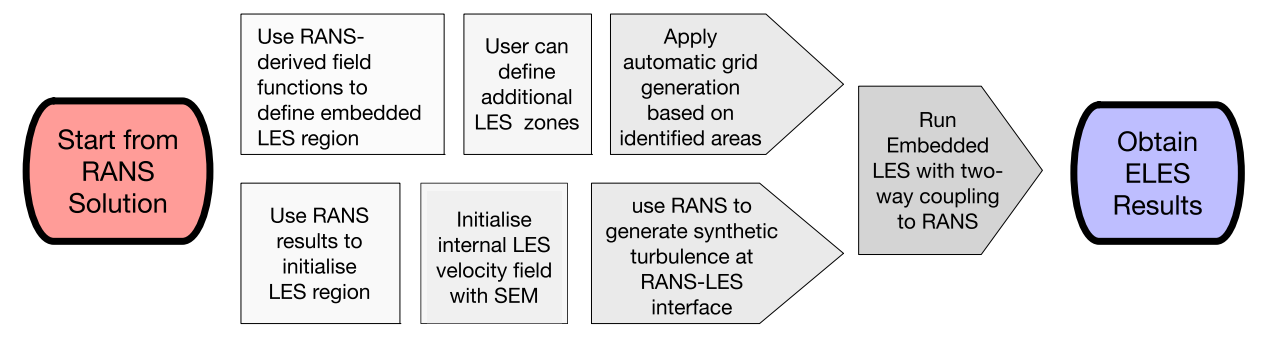

reduced. Not only does this enable reduced workflow times, but it also increases simulation repeatability, which in many ways is of greater importance than overall simulation accuracy; since in many industrial CFD studies it is the qualitative information which is important.

Figure 9 illustrates the process for which the automated system could follow. This process automatically implements the general capabilities outlined by Cokljat et al. [14] for which an ELES model is required to provide in an industrial environment. A pre-cursor RANS calculation allows for sufficient knowledge of the flow-field to be obtained, so that a suitable LES mesh can be generated and the extent of regions requiring further detailed simulation can be identified. The overall aim of automation is to allow for a streamlined process chain for computing internal fluid flow. Such a process remains to be clearly defined although preliminary work by Prost et al. [76] has demonstrated the concept of automated RANS/LES interface control by using algebraic sensors to and distinguish between attached and detached flow regions.

\subsection{Multi-Dimensional Coupling}

Finally we note that in many industrial sectors the need to retain low spatial dimension simulations will likely remain for some time. This is particularly relevant for systems where the flow length scale, e.g. pipe diameter, is much smaller than the overall system dimension e.g. pipe length. For example, gas network companies who consider flow analysis over vast lengths of pipelines simply cannot afford full 3D or even 2D simulation of the whole system; and in most instances 1D modelling is sufficient. However there are invariably instances where more detailed simulation is required and hence the industry must step from $1 \mathrm{D}$ to $3 \mathrm{D}$ analysis. The idea of multi-dimensional coupling involves a continuous workflow or seamless transition between differing physical platforms could be achieved. This is portrayed in Fig. 10 which illustrates a transition from fast 1D interactive tools to high-detail 3D complex physics tools. For the analysis of pipe networks the 1D studies can be used to model flow through an expansive network, and to quickly cycle through a range of scenarios in order to identify the critical cases. A subset of these cases could then be studied in 3D, combining RANS with LES where needed; for example to provide unsteady $3 \mathrm{D}$ insight to a range of piecewise components or larger combined scenarios (such as bends, valves and compressors) to examine the detailed impact of surges through precise representation of local pipework and equipment. For more details see [35].

This concept is already receiving interest. A recently coupled 1D - 3D solver implemented in OpenFOAM uses Riemann invariants to ensure the 1D boundaries are updated each time step from the computed 3D region, whilst the 3D inlet boundaries apply the upstream 1D pressure head for the pressure whilst the velocity is set from a homogenous Neumann condition; this method was successfully applied to transient pipe flows with only minor losses noted [121]. In addition, an earlier publication by Prince [75] focussed on the coupling of 1D and 3D systems for computing flows through subway transit networks.

\section{Conclusion}

It has been demonstrated that hybrid RANS-LES approaches have a role to play within industry to facilitate scale resolution of turbulent flows. Furthermore the benefits of embedded LES, where reduced computational costs are clear compared to full LES, whilst providing an equivalent level of fidelity within chosen regions of the domain. This paper has established the feasibility of applying hybrid RANS-LES methods to industrial CFD applications, whilst documenting the separate ingredients involved within the 
Fig. 10 Concept for multidimensional coupling process for pipe networks (top), mesh used for 1D-3D of water hammer in pipes from [121]
1D

2D

3D

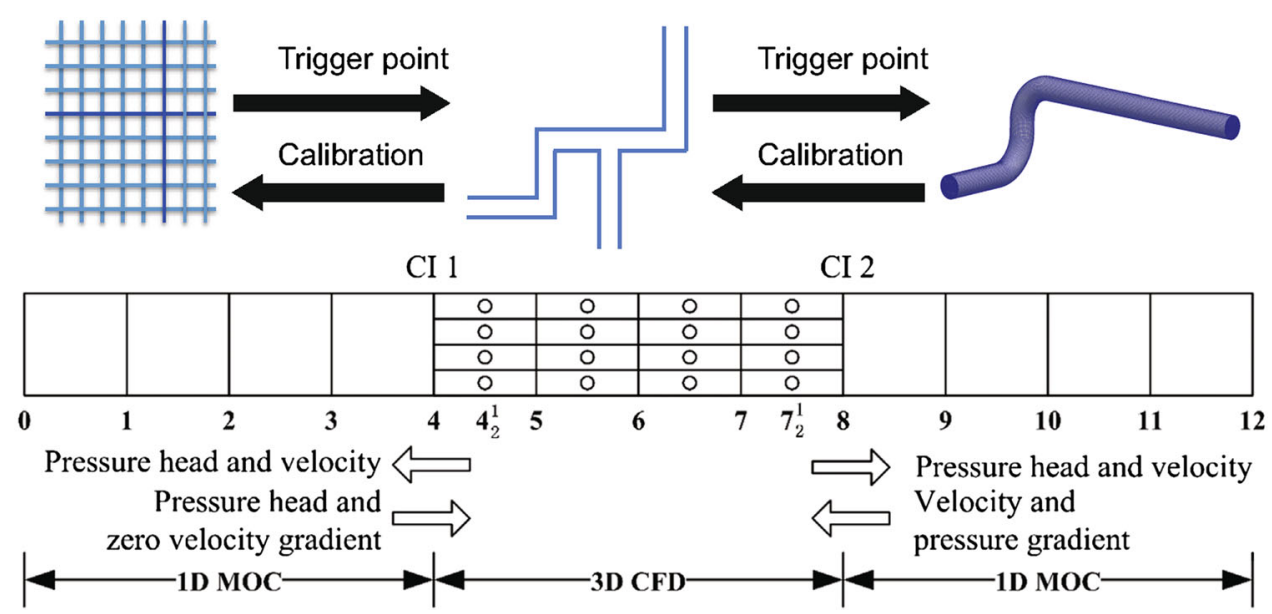

approaches. It is clear that the use of a hybrid solver would allow for a reduction in total grid resolution over traditional LES, thus reducing both the computational expense and simulation time, whilst also potentially increasing the overall simulation accuracy when compared to RANS.

Future trends have been discussed and greater efficiency and repeatability could be attained by automating the computational process when introducing the two concepts outlined above. For example by reducing user input required to generate suitable meshes for turbulence simulation and making use of synthetic turbulence generators to pass from RANS to LES regions. The development of multi-dimensional coupling methods is particularly useful for industries which consider pipelines or systems which operate over a significant separation of scales.

Acknowledgements This work has been supported primarily by an iCASE award from National Grid Gas. We also include some simulation results supported by the UK Turbulence Consortium (EPSRC Grant EP/G069581/1), which provided computational access to ARCHER, the UKs national high-performance computing service. We also made some use of the facilities at N8 HPC, provided and funded by the N8 consortium and EPSRC (Grant No. EP/K000225/1).

\section{Compliance with Ethical Standards}

Conflict of interest The authors declare that they have no conflict of interest.

Open Access This article is distributed under the terms of the Creative Commons Attribution 4.0 International License (http://creative commons.org/licenses/by/4.0/), which permits unrestricted use, distribution, and reproduction in any medium, provided you give appropriate credit to the original author(s) and the source, provide a link to the Creative Commons license, and indicate if changes were made.

\section{References}

1. Adamian DY, Travin AK (2011) Improved version of the synthetic eddy method for setting nonstationary inflow boundary conditions in calculating turbulent flows. High Temp 49(5):704-711. https://doi.org/10.1134/S0018151X11040018

2. Anupindi K, Sandberg R (2016) Implementation and evaluation of an embedded LES-RANS solver. Flow Turbul Combust. https://doi.org/10.1007/s10494-016-9787-5

3. Aounallah M, Belkadi M, Adjlout L, Imine O (2013) RANS simulations of turbulent and thermal mixing in a T-junction. Mechanika 19(3):277-282

4. Arvidson S (2013) Assessment and some improvements of hybrid RANS-LES methods. Ph.D. thesis

5. Auerswald T, Bange J (2016) A new method to generate anisotropic synthetic turbulence for LES. Adv Simul Wing Nacelle Stall 131:223-233. https://doi.org/10.1007/978-3-319-21127-5

6. Auerswald T, Probst A, Bange J (2016) An anisotropic synthetic turbulence method for large-eddy simulation. Int J Heat Fluid Flow 62:407-422. https://doi.org/10.1016/j.ijheatfluidflow.2016. 09.002

7. Aulery F, Toutant A, Monod R, Brillant G, Bataille F (2012) Numerical simulations of sodium mixing in a T-junction. Appl Therm Eng 37:38-43. https://doi.org/10.1016/j.applthermaleng. 2011.12.044

8. Basara B, Krajnovic S, Girimaji S, Pavlovic Z (2011) Near-wall formulation of the partially averaged Navier Stokes turbulence model. AIAA J 49(12):2627-2636

9. Casarsa L, Cakan M, Arts T (2002) Characterization of the velocity and heat transfer fields in an internal coooling channel with high blockage ratio. In: Proceedings of the ASME turbo exposition

10. Chaouat B, Schiestel R (2005) A new partially integrated transport model for subgrid-scale stresses and dissipation rate for turbulent developing flows. Phys Fluids 17(6):065106. https://doi.org/10.1063/1.1928607

11. Chapman DR (1979) Computational aerodynamics development and outlook. AIAA J 17(12):1293-1313

12. Chapuliot S, Gourdin C, Payen T, Magnaud J, Monavon A (2005) Hydro-thermal-mechanical analysis of thermal fatigue in a mixing tee. Nucl Eng Des 235(5):575-596. https://doi.org/10. 1016/j.nucengdes.2004.09.011 
13. Choi H, Moin P (2012) Grid-point requirements for large eddy simulation: Chapman's estimates revisited. Phys Fluids. https:// doi.org/10.1063/1.3676783

14. Cokljat D, Caridi D, Link G, Lechner R, Menter F (2009) Embedded LES methodology for general-purpose CFD solvers. In: 6th international symposium on turbulence and shear flow phenomena, pp 1191-1196

15. Davidson L (2000) LES-RANS of channel flow (April). Chalmers University of Technology, Göteborg, Sweden

16. Davidson L (2006) Evaluation of the SST-SAS model: channel flow, asymmetric diffuser and axisymmetric hill. In: Proceedings of the European conference on computational fluid dynamics (ECCOMAS CFD 2006), pp 1-20

17. Davidson L (2014) The PANS model in a zonal hybrid RANSLES formulation. Int $\mathrm{J}$ Heat Fluid Flow 46:112-126. https://doi.org/10.1016/j.ijheatfluidflow.2014.01.002

18. Davidson L, Peng SH (2013) Embedded large-eddy simulation using the partially averaged Navier-Stokes model. AIAA J 51:1066-1079

19. De Laage de Meux B (2012) Modélisation des écoulements turbulents en rotation et en présence de transferts thermiques par approche hybride RANS/LES zonale. Ph.D. thesis, École Nationale Supérieure de Mécanique et d'Aérotechnique

20. de Laage de Meux B, Audebert B, Manceau R, Perrin R (2015) Anisotropic linear forcing for synthetic turbulence generation in large eddy simulation and hybrid RANS/LES modeling. Phys Fluids 27(3):035115. https://doi.org/10.1063/1.4916019

21. Dean WR, Hurst JM (1927) Note on the motion of fluid in a curved pipe. Philos Mag 4(20):208-223. https://doi.org/10.1112/ S0025579300001947

22. Deck S (2005) Numerical simulation of transonic buffet over a supercritical airfoil. AIAA J 43(7):1556-1566. https://doi.org/ $10.2514 / 1.9885$

23. Deck S (2005) Zonal detached-eddy simulation of the flow around a high-lift configuration with deployed slat and flap. AIAA J 43:2372-2384

24. Deck S (2011) Recent improvements in the zonal detached eddy simulation (ZDES) formulation. Theor Comput Fluid Dyn 26(6):523-550. https://doi.org/10.1007/s00162-011-0240-z

25. Deck S, Weiss PE, Pamies M, Garnier E (2008) On the use of stimulated detached eddy simulation (SDES) for spatially developing boundary layers. Notes Numer Fluid Mech Multidiscip Des 97(1998):67-76. https://doi.org/10.1007/978-3-54077815-87

26. Deck S, Weiss PÉ, Pamiès M, Garnier E (2011) Zonal detached eddy simulation of a spatially developing flat plate turbulent boundary layer. Compute Fluids 48(1):1-15. https://doi.org/10. 1016/j.compfluid.2011.03.009

27. Frank T, Lifante C, Prasser HM, Menter F (2010) Simulation of turbulent and thermal mixing in T-junctions using URANS and scaleresolving turbulence models in ANSYS CFX. Nucl Eng Des 240(9):2313-2328. https://doi.org/10.1016/j.nucengdes.2009.11.008

28. Fureby C (2017) Challenges for large eddy simulation of engineering flows. In: Pollard A, Castillo L, Danaila L, Glauser M (eds) Whither turbulence and big data in the 21st century? Chap. 20. Springer International Publishing, Berlin, pp 375-400

29. Gauder P, Karthick Selvam P, Kulenovic R, Laurien E (2016) Large eddy simulation studies on the influence of turbulent inlet conditions on the flow behavior in a mixing tee. Nucl Eng Des 298(May 1998):51-63. https://doi.org/10.1016/j.nucengdes.2015. 12.001

30. Girimaji S, Abdol-Hamid K (2005) Partially averaged Navier Stokes model for turbulence: implementation and validation. In: AIAA paper (January), pp 1-14

31. Gritskevich M, Garbaruk A, Frank T, Menter F (2014) Investigation of the thermal mixing in a $\mathrm{T}$-junction flow with different
SRS approaches. Nucl Eng Des 279:83-90. https://doi.org/10. 1016/j.nucengdes.2014.03.010

32. Gritskevich M, Garbaruk A, Frank T, Menter FR (2012) Investigation of the thermal mixing in a $\mathrm{T}$-junction flow with different SRS approaches. In: Proceedings of CFD4NRS-4 the experimental validation and application of CFD and CMFD codes in nuclear reactor technology, pp 1-11

33. Gritskevich MS, Garbaruk AV, Menter FR (2014) Computation of wall bounded flows with heat transfer in the framework of SRS approaches. J Phys Conf Ser 572:012057. https://doi.org/ 10.1088/1742-6596/572/1/012057

34. Gritskevich MS, Garbaruk AV, Menter FR (2016) Investigation of the passage between LES and RANS subdomains in the framework of zonal RANS-LES approaches. J Phys Conf Ser 769:012080. https://doi.org/10.1088/1742-6596/769/1/012080

35. Holgate J, Skillen A, Revell A, Craft T (2016) Application of embedded simulation to internal flows. In: United Kingdom turbulence consortium

36. Hoyas S, Jiménez J (2006) Scaling of the velocity fluctuations in turbulent channels up to $\operatorname{Re} \tau=2003$. Phys Fluids 18(1):10-14. https://doi.org/10.1063/1.2162185

37. Jakirlić S, Manceau R, Šarić S, Fadai-Ghotbi A, Kniesner B, Carpy S, Kadavelil G, Friess C, Tropea C, Borée J (2009) LES, zonal and seamless hybrid LES/RANS: rationale and application to free and wall-bounded flows involving separation and swirl. In: Brun $\mathrm{C}$ et al (eds) Numerical simulation of turbulent flows and noise generation. Springer, Berlin, pp 253-282

38. Jarrin N, Benhamadouche S (2006) A synthetic-eddy-method for generating inflow conditions for large-eddy simulations. Int $\mathrm{J}$ Heat Fluid Flow 27:585-593. https://doi.org/10.1016/j.ijheat fluidflow.2006.02.006

39. Jarrin N, Prosser R, Uribe JC, Benhamadouche S, Laurence D (2009) Reconstruction of turbulent fluctuations for hybrid RANS/LES simulations using a synthetic-eddy method. Int $\mathrm{J}$ Heat Fluid Flow 30(3):435-442. https://doi.org/10.1016/j.ijheat fluidflow.2009.02.016

40. Jasak H, Weller HG, Gosman AD (1999) High resolution NVD differencing scheme for arbitararily unstructured meshes. Int $\mathbf{J}$ Numer Method Fluids 31:431-449

41. Jørgensen N, Kossa H, Bennetsenb J (2012) Embedded-LES and experiment of turbulent boundary layer flow around a floormounted cube. In: The Seventh international colloquium, pp 224-233

42. Jørgensen NG, Koss H, Bennetsen J (2012) Embedded-LES and experiment of turbulent boundary layer flow around a floormounted cube . In: The seventh international colloqium on bluff body aerodynamics and applications

43. Kalitzin G, Medic G, Templeton JA (2008) Wall modeling for LES of high Reynolds number channel flows: What turbulence information is retained? Comput Fluids 37(7):809-815. https:// doi.org/10.1016/j.compfluid.2007.02.016

44. Kamali R, Binesh A (2008) The importance of rib shape effects on the local heat transfer and flow friction characteristics of square ducts with ribbed internal surfaces. Int Commun Heat Mass Transf 35(8):1032-1040. https://doi.org/10.1016/j.icheat masstransfer.2008.04.012

45. Kapulla R, Mignot G, Paranjape S, Ryan L, Paladino D (2014) Large scale gas stratification erosion by a vertical helium-air jet. Sci Technol Nucl Install. https://doi.org/10.1155/2014/197267

46. König D, Meinke M, Schröder W (2010) Embedded LES-toRANS boundary in zonal simulations. J Turbul 11(January 2015):N7. https://doi.org/10.1080/14685241003698159

47. Kubacki S, Rokicki J, Dick E (2014) Hybrid RANS/LES of flow in a rib-roughened rotating channel. Proc ASME Turbo Expo 2014:1-12 
48. Kuczaj AK, Komen E, Loginov M (2010) Large-eddy simulation study of turbulent mixing in a T-junction. Nucl Eng Des 240(9):2116-2122. https://doi.org/10.1016/j.nucengdes.2009.11. 027

49. Labbé $O$ (2013) Large-eddy-simulation of flow and heat transfer in a ribbed duct. Comput Fluids 76:23-32. https://doi.org/10. 1016/j.compfluid.2013.01.023

50. Laraufie R, Deck S, Sagaut P (2011) A dynamic forcing method for unsteady turbulent inflow conditions. J Comput Phys 230(23):8647-8663. https://doi.org/10.1016/j.jcp.2011.08.012

51. Lardeau S, Manceau R (2014) Computations of canonical and complex flow configurations using a robust formulation of the elliptic-blending Reynolds-stress model to cite this version: using a modified elliptic-blending Reynolds-stress model. In: 10th engineering turbulence modelling and measurement conference (ETMM10)

52. Larsson J, Wang Q (2014) The prospect of using LES and DES in engineering design, and the research required to get there. R Soc Philos Trans A 372:1-15

53. Lee JI, Hu Lw, Saha P, Kazimi MS (2009) Numerical analysis of thermal striping induced high cycle thermal fatigue in a mixing tee. Nucl Eng Des 239(5):833-839. https://doi.org/10. 1016/j.nucengdes.2008.06.014

54. Li Z, Chen H, Zhang Y (2016) Validation of a window-embedded RANS/LES method based on synthetic turbulence. In: 54th AIAA aerospace sciences meeting (January), pp 1-8. https://doi.org/10.2514/6.2016-0339

55. Lin C, Ferng Y (2016) Investigating thermal mixing and reverse flow characteristics in a T-junction using CFD methodology. Appl Therm Eng 102:733-741. https://doi.org/10.1016/j.appl thermaleng.2016.03.124

56. Liu Y, Tucker PG, Lo Iacono G (2006) Comparison of zonal RANS and LES for a non-isothermal ribbed channel flow. Int $\mathrm{J}$ Heat Fluid Flow 27(3):391-401. https://doi.org/10.1016/j.ijheat fluidflow.2005.11.004

57. Lohász MM, Rambaud P, Benocci C (2006) Flow features in a fully developed ribbed duct flow as a result of MILES. Flow Turbul Combust 77(1-4):59-76. https://doi.org/10.1007/s10494006-9037-3

58. Mathey F, Cokljat D, Bertoglio JP, Sergent E (2006) Assessment of the vortex method for large eddy simulation inlet conditions. Prog Comput Fluid Dyn Int J 6:58-67

59. Menter FR, Kuntz M (2004) Adaptation of eddy-viscosity turbulence models to unsteady turbulence models to unsteady separated flow behind vehicles. Aerodyn Heavy Veh 19:339-352

60. Menter FR, Schütze J, Gritskevich M (2012) Global vs. zonal approaches in hybrid RANS-LES turbulence modelling. In: Fu S, Haase W, Peng SH, Schamborn D (eds) Progress in hybrid RANS-LES modelling. Notes on numerical fluid mechanics and multidisciplinary design, vol 117. Springer, Berlin

61. Ming T, Zhao J (2012) Large-eddy simulation of thermal fatigue in a mixing tee. Int J Heat Fluid Flow 37:93-108. https://doi.org/ 10.1016/j.ijheatfluidflow.2012.06.002

62. Ndombo JM, Howard RJ (2011) Large eddy simulation and the effect of the turbulent inlet conditions in the mixing tee. Nucl Eng Des 241(6):2172-2183. https://doi.org/10.1016/j. nucengdes.2011.03.020

63. Obabko AV (2011) CFD Validation in OECD/NEA T junction benchmark. Technical report

64. Ooi A, Iaccarino G, Durbin P, Behnia M (2002) Reynolds averaged simulation of flow and heat transfer in ribbed ducts. Int J Heat Fluid Flow 23(6):750-757. https://doi.org/10.1016/ S0142-727X(02)00188-1

65. Ould-Rouiss M, Bousbai M, Mazouz A (2013) Large eddy simulation of turbulent heat transfer in pipe flows with respect to
Reynolds and Prandtl number effects. Acta Mech 224(5):1133-1155. https://doi.org/10.1007/s00707-012-0796-8

66. Pamies M, Weiss PE, Garnier E, Deck S, Sagaut P (2009) Generation of synthetic turbulent inflow data for large eddy simulation of spatially evolving wall-bounded flows. Phys Fluids 21(4):045103. https://doi.org/10.1063/1.3103881

67. Pantano C, Pullin DI, Dimotakis PE, Matheou G (2008) LES approach for high Reynolds number wall-bounded flows with application to turbulent channel flow. J Comput Phys 227(21):9271-9291. https://doi.org/10.1016/j.jcp.2008.04.015

68. Pasutto T, Peniguel C, Stephan JM (2007) Effect of the upstream elbows for thermal fatigue studies of PWR T-junction using large eddy simulations. In: 15th international conference on nuclear engineering, pp 1-8

69. Patruno L, Ricci M (2017) On the generation of synthetic divergence-free homogeneous anisotropic turbulence. Comput Methods Appl Mech Eng 315:396-417. https://doi.org/10.1016/ j.cma.2016.11.005

70. Piomelli U (2008) Wall-layer models for large-eddy simulations. Prog Aerosp Sci 44(6):437-446

71. Poletto R, Revell A, Craft T, Jarrin N (2011) Divergence free sythentic eddy method for embedded LES inflow boundary conditions. In: Seventh international symposium on turbulence and shear flow phenomena

72. Poletto R, Revell A, Craft T, Ashton N (2012) Embedded DDES of 2D hump flow. Notes Numer Fluid Mech Multidiscip Des 117:169-179. https://doi.org/10.1007/978-3-642-31818-4-15

73. Poletto R, Craft T, Revell A (2013) A new divergence free synthetic eddy method for the reproduction of inlet flow conditions for LES. Flow Turbul Combust 91(3):519-539. https:// doi.org/10.1007/s10494-013-9488-2

74. Pope SB (2000) Turbulent flows. Cambridge University Press, Cambridge

75. Prince JA (2015) Coupled 1D-3D simulation of flow in subway transit networks. Ph.D. thesis, Imperial College, London

76. Probst A, Radespiel R, Knopp T (2011) Detached-eddy simulation of aerodynamic flows using a Reynolds-stress background model and algebraic RANS/LES sensors. In: 20th AIAA computational fluid dynamics conference, pp 1-20

77. Röhrig R, Jakirlić S, Tropea C (2015) Comparative computational study of turbulent flow in a 90 pipe elbow. Int J Heat Fluid Flow 55:120-131. https://doi.org/10.1016/j.ijheatfluidflow.2015. 07.011

78. Schlüter JU, Pitsch H, Moin P (2004) LES inflow conditions for coupling with Reynolds averaged flow solvers. AIAA J. https:// doi.org/10.2514/1.3488

79. Schluter JU, Wu X, Kim S, Shankaran S, Alonso JJ, Pitsch H (2005) A framework for coupling Reynolds-averaged with large-eddy simulations for gas turbine applications. J Fluids Eng 127:806. https://doi.org/10.1115/1.1994877

80. Schuler M, Dreher HM, Neumann SO, Weigand B, Elfert M (2012) Numerical predictions of the effect of rotation on fluid flow and heat transfer in an engine-similar two-pass internal cooling channel with smooth and ribbed walls. J Turbomach 134(2):021021. https://doi.org/10.1115/1.4003086

81. Sergent E (2002) Vers une méthodologie de couplage entre la simulation des grandes echelles et les modèles statistiques. Ph.D. thesis, Ecole Central de Lyon

82. Sewall EA, Tafti DK (2006) Large eddy simulation of flow and heat transfer in the 180-deg bend region of a stationary gas turbine blade ribbed internal cooling duct. J Turbomach 128(4):763. https://doi.org/10.1115/1.2098769

83. Sewall EA, Tafti DK (2008) Large eddy simulation of flow and heat transfer in the developing flow region of a rotating gas turbine blade internal cooling duct with coriolis and buoyancy 
forces. J Turbomach 130(1):011005. https://doi.org/10.1115/1. 2437779

84. Sewall EA, Tafti DK, Graham AB, Thole KA (2006) Experimental validation of large eddy simulations of flow and heat transfer in a stationary ribbed duct. Int $\mathbf{J}$ Heat Fluid Flow 27(2):243-258. https://doi.org/10.1016/j.ijheatfluidflow.2005. 08.010

85. Shawish A, Salama M (2014) Cloud computing: paradigms and technologies. In: Fatos Xhafa NB (ed) Inter-cooperative collective intelligence: techniques and applications, 1st edn. Springer, Berlin, pp 39-67

86. Shur ML, Spalart PR, Strelets MK, Travin AK (2008) A hybrid RANS-LES approach with delayed-DES and wall-modelled LES capabilities. Int J Heat Fluid Flow 29(6):1638-1649. https://doi.org/10.1016/j.ijheatfluidflow.2008.07.001

87. Shur ML, Spalart PR, Strelets MK, Travin AK (2014) Synthetic turbulence generators for RANS-LES interfaces in zonal simulations of aerodynamic and aeroacoustic problems. Flow Turbul Combust 93(1):63-92. https://doi.org/10.1007/s10494-0149534-8

88. Skillen A, Revell A, Craft T (2016) Accuracy and efficiency improvements in synthetic eddy methods. Int J Heat Fluid Flow. https://doi.org/10.1016/j.ijheatfluidflow.2016.09.008

89. Smagorinsky J (1963) General ciculation experiments with the primitive equations. Mon Weather Rev 91(3):99-164

90. Sohankar A (2010) Heat transfer and fluid flow through a ribbed passage in staggered arrangement. Iran $\mathrm{J}$ Sci Technol 34:471-485

91. Spalart P (2000) Strategies for turbulence modelling and simulations. Int J Heat Fluid Flow 21(3):252-263. https://doi.org/10. 1016/S0142-727X(00)00007-2

92. Spalart PR, Allmaras SR (1994) A one-equation turbulence model for aerodynamic flows. Recherche Aerospatiale, No. 1, pp 5-21

93. Spalart PR, Deck S, Shur ML, Squires KD, Strelets MK, Travin A (2006) A new version of detached-eddy simulation, resistant to ambiguous grid densities. Theor Comput Fluid Dyn 20(3):181-195. https://doi.org/10.1007/s00162-006-0015-0

94. Spalart PR, Jou WH, Strelets MK, Allmaras SR (1997) Comments on the feasibility of LES for wings, and on a hybrid RANS/LES approach. In: International conference on LES/ DNS, pp 137-147

95. Strelets M (2001) Detached eddy simulation of massively separated flows. In: 39th Aerospace sciences meeting and exhibit, p 879

96. Tabor G, Baba-Ahmadi M (2010) Inlet conditions for large eddy simulation: a review. Comput Fluids 39(4):553-567. https://doi. org/10.1016/j.compfluid.2009.10.007

97. Tafti D (2005) Evaluating the role of subgrid stress modeling in a ribbed duct for the internal cooling of turbine blades. Int $\mathrm{J}$ Heat Fluid Flow 26(1):92-104. https://doi.org/10.1016/j.ijheatfluid flow.2004.07.002

98. Takamura H, Ebara S, Kaneko T, Yamano H, Hashizume H (2012) Flow structure of high Reynolds number flow in a dualelbow piping simulation cold-legs of JSFR. In: 15th international symposium on flow visualization, pp 25-28

99. Tanaka M, Ohshima H (2012) Numerical investigation on large scale eddy structure in unsteady pipe elbow flow at high Reynolds number conditions with large eddy simulation approach. J Power Energy Syst 6(2):210-228. https://doi.org/10.1299/jpes. 6.210

100. Tavakoli E, Hosseini R, Papalexandris M, Lessani B (2013) Statistical analysis of instantaneous turbulent heat transfer in circular pipe flows. Heat Mass Transf 50(1):125-137. https:// doi.org/10.1007/s00231-013-1230-z
101. Temmermann L, Leschziner MA, Hanjalic K (2002) A-priori studies of a near-wall RANS model within a hybrid LES/RANS scheme. In: Rodi W, Fueyo N (eds) Engineering turbulence modelling and experiments, vol 5. Elsevier, Amsterdam, pp 317-326

102. Tucker $P$ (2011) Computation of unsteady turbomachinery flows: part 2 LES and hybrids. Prog Aerosp Sci 47(7):546-569. https://doi.org/10.1016/j.paerosci.2011.07.002

103. Tucker $P$ (2013) Large eddy simulation of turbine internal cooling ducts. In: Proceedings of the ASME 2013 international mechanical engineering congress and exposition, pp 1-13

104. Tucker P (2013) Trends in turbomachinery turbulence treatments. Prog Aerosp Sci 63:1-32

105. Tucker P, Davidson L (2004) Zonal kl based large eddy simulations. Comput Fluids 33(2):267-287. https://doi.org/10.1016/ S0045-7930(03)00039-2

106. Tunstall R, Laurence D, Prosser R, Skillen A (2016) Benchmarking LES with wall-functions and RANS for fatigue problems in thermalhydraulics systems. Nucl Eng Des 308:170-181. https://doi.org/10.1016/j.nucengdes.2016.08.022

107. Tunstall R, Laurence D, Prosser R, Skillen A (2016) Large eddy simulation of a T-junction with upstream elbow: the role of Dean vortices in thermal fatigue. Appl Therm Eng 107:672-680. https://doi.org/10.1016/j.applthermaleng.2016.07.011

108. Tunstall R, Laurence D, Prosser R, Skillen A (2017) Towards a generalised dual-mesh hybrid LES/RANS framework with improved consistency. Comput Fluids. https://doi.org/10.1016/j. compfluid.2017.08.002

109. Tyacke J, Tucker P (2015) Large eddy simulation of turbine internal cooling ducts. Comput Fluids 114:130-140. https://doi. org/10.1016/j.compfluid.2015.02.022

110. Uribe JC, Revell A, Moulinec C (2010) Computation of flow in a 3D diffuser using a two-velocity field hybrid RANS/LES. Notes Numer Fluid Mech Multidiscip Des 110:385-391. https:// doi.org/10.1007/978-3-642-14139-3-47

111. Viswanath R, Group M, Corp I (2000) Thermal performance challenges from silicon to systems. Intel Technol J Q3:1-16

112. Viswanathan AK (2006) Detached eddy simulation of turbulent flow and heat transfer in turbine blade internal cooling ducts. Ph.D. dissertation, Virginia Polytechnic Institute and State University, Blacksburg, VA

113. Viswanathan AK, Tafti DK (2005) Detached eddy simulation of turbulent flow and heat transfer in a ribbed duct. J Fluids Eng 127(5):888. https://doi.org/10.1115/1.2033010

114. Viswanathan AK, Tafti DK (2006) A comparative study of DES and URANS for flow prediction in a two-pass internal cooling duct. J Fluids Eng 128(6):1336. https://doi.org/10.1115/1. 2353279

115. Viswanathan AK, Tafti DK (2006) Detached eddy simulation of flow and heat transfer in fully developed rotating internal cooling channel with normal ribs. Int $\mathrm{J}$ Heat Fluid Flow 27(3):351-370. https://doi.org/10.1016/j.ijheatfluidflow.2005. 12.003

116. Viswanathan AK, Tafti DK (2006) Detached eddy simulation of turbulent flow and heat transfer in a two-pass internal cooling duct. Int J Heat Fluid Flow 27(1):1-20. https://doi.org/10.1016/j. ijheatfluidflow.2005.07.002

117. Viswanathan AK, Tafti DK (2007) Investigation of detached eddy simulations in capturing the effects of coriolis forces and centrifugal buoyancy in ribbed ducts. J Heat Transf 129(7):778. https://doi.org/10.1115/1.2717944

118. Vonlanthen M, Allegrini J, Carmeliet J (2016) Assessment of a one-way nesting procedure for obstacle resolved large eddy simulation of the ABL. Comput Fluids 140:136-147. https://doi. org/10.1016/j.compfluid.2016.09.016 
119. Waldrop M (2016) The chips are down for Moore's law. Nat Int Wkly J Sci 530:144-147

120. Walker C, Manera A, Niceno B, Simiano M, Prasser HM (2010) Steady-state RANS-simulations of the mixing in a T-junction. Nucl Eng Des 240(9):2107-2115. https://doi.org/10.1016/j. nucengdes.2010.05.056

121. Wang C, Nilsson H, Yang J, Petit O (2017) 1D3D coupling for hydraulic system transient simulations. Comput Phys Commun 210:1-9. https://doi.org/10.1016/j.cpc.2016.09.007

122. Westin J, Alavyoon F, Andersson L, Veber P, Henriksson M, Andersson C (2006) Experiments and Unsteady CFD-Calculations of Thermal Mixing in a T-junction. In: Proceedings of OECD/NEA/IAEA workshop on the benchmarking of CFD codes for application to nuclear reactor safety (CFD4NRS), Munich Germany, vol 1,pp 1-15

123. Wu D, Rosen DW, Wang L, Schaefer D (2015) Cloud-based design and manufacturing: a new paradigm in digital manufacturing and design innovation. CAD Comput Aided Des 59:1-14. https://doi.org/10.1016/j.cad.2014.07.006

124. Wu X (2017) Inflow turbulence generation methods. Annu Rev Fluid Mech 49(1):23-49. https://doi.org/10.1146/annurev-fluid010816-060322
125. Xiao H, Jenny P (2012) A consistent dual-mesh framework for hybrid LES/RANS modeling. J Comput Phys 231(4):1848-1865. https://doi.org/10.1016/j.jcp.2011.11.009

126. Xie G, Li S, Zhang W, Sunden B (2013) Computational fluid dynamics modeling flow field and side-wall heat transfer in rectangular rib-roughened passages. J Energy Resour Technol 135(4):042001. https://doi.org/10.1115/1.4023332

127. Yuki K, Hasegawa S, Sato T, Hashizume H, Aizawa K, Yamano H (2011) Matched refractive-index PIV visualization of complex flow structure in a three-dimentionally connected dual elbow. Nucl Eng Des 241(11):4544-4550. https://doi.org/10. 1016/j.nucengdes.2010.12.026

128. Zeng C, Li CW (2010) A hybrid RANS-LES model for flows in open channel T-diversions. In: 9th international conference on hydrohynamics, pp 1-10

129. Zhang T, Zhang Y, Ouyang H (2015) Structural vibration and fluid-borne noise induced by turbulent flow through a 90 piping elbow with/without a guide vane. Int J Press Vessels Pip 125:66-77. https://doi.org/10.1016/j.ijpvp.2014.09.004 